\title{
Inclusion of the Acetabular Labrum Reduces Simulated Range of Motion of the Hip Compared With Bone Contact Models
}

\author{
Penny R. Atkins, Ph.D., Takehito Hananouchi, M.D., Ph.D., Andrew E. Anderson, Ph.D., \\ and Stephen K. Aoki, M.D.
}

\begin{abstract}
Purpose: To determine whether inclusion of the acetabular labrum affects the maximum range of motion (ROM) during simulation of the flexion-adduction-internal rotation impingement examination. Methods: Three-dimensional surface reconstructions of the femur, hemi-pelvis, and labrum from computed tomography arthrography images of 19 participants were used to simulate maximum ROM during the flexion-adduction-internal rotation examination. Simulations were conducted for positions between $70^{\circ}$ and $110^{\circ}$ flexion and $0^{\circ}$ and $20^{\circ}$ adduction at $10^{\circ}$ increments to measure maximum internal rotation and the position of contact between the femur and acetabular rim (bone-to-bone) or the femur and labrum (bone-to-labrum). Internal rotation angles and clock-face position values were compared between the 2 contact scenarios for each position. Results: The ROM in the bone-to-labrum contact model was significantly less than that of the bone-to-bone contact model for all evaluated positions $\left(P \leq .001\right.$, except at $110^{\circ}$ flexion and $20^{\circ}$ adduction, $\left.P=.114\right)$. The inclusion of the labrum reduced internal rotation by a median [interquartile range] of $18[15,25]^{\circ}$ while altering the position of contact on the acetabular clock-face by $-0: 01[-0: 27,0: 16]$. The variability in contact location for the bone-to-labrum contact scenario was nearly double that of the bone-to-bone contact scenario, as indicated by the interquartile range. Conclusions: Inclusion of the anatomy of the acetabular labrum in collision models used to simulate impingement examinations reduced the internal rotation ROM by approximately $20^{\circ}$ and increased variability in the location of contact relative to the acetabular rim. Clinical Relevance: While standard bone-to-bone contact ROM simulations may be informative with respect to the relative change in ROM based on a surgical intervention (e.g., pre- and post-osteochondroplasty for cam-type femoroacetabular impingement), they may not accurately represent the clinical ROM of the joint or the kinematic position at which damage may occur due to shape mismatch between the femur and acetabulum.
\end{abstract}

$\mathbf{F}$ emoroacetabular impingement (FAI) is described as atypical abutment between the femur and acetabulum that occurs at relatively low ranges of motion (ROMs) and results in reduced ROM, pain, and disability. ${ }^{1}$ The

From the Departments of Orthopaedics (P.R.A., T.H., A.E.A., S.K.A.), Bioengineering (P.R.A., A.E.A.), and Physical Therapy (A.E.A.), University of Utah, Salt Lake City, Utah, U.S.A.; and Medical Engineering Laboratory, Department of Mechanical Engineering, Osaka Sangyo University, Daito, Osaka, Japan (T.H.)

The authors report the following potential conflicts of interest or sources of funding: P.R.A. reports grants from the National Institutes of Health (NIH) and grants from LS Peery Discovery Program in Musculoskeletal Research, during the conduct of this study. T.H. reports grants from LS Peery Discovery Program in Musculoskeletal Research, during the conduct of this study. A.E.A. reports grants from the NIH and grants from LS Peery Discovery Program in Musculoskeletal Research, during the conduct of this study. S.K.A. reports grants from the NIH and grants from LS Peery Discovery Program in Musculoskeletal Research, during the conduct of this study, and other from Stryker, outside the submitted work. The authors acknowledge funding from the NIH (R21AR063844 and S1ORR026565) and the LS Peery Discovery Program in Musculoskeletal Restoration. The research abnormal joint contact associated with cam-type FAI is a result of asphericity of the femoral head, whereas pincertype FAI is due to overcoverage of the femoral head by the acetabulum. Both types of FAI are recognized clinically by

content herein is solely the responsibility of the authors and does not necessarily represent the official views of the NIH or LS Peery Discovery Program. Full ICMJE author disclosure forms are available for this article online, as supplementary material.

Penny R. Atkins, Ph.D., and Takehito Hananouchi, M.D., contributed equally to this article.

Received March 4, 2020; accepted July 16, 2020.

Address correspondence to Stephen K. Aoki, University of Utah Orthopaedics, 590 Wakara Way, Salt Lake City,UT84108.E-mail: stephen.aoki@ hsc.utah.edu

(C) 2020 THE AUTHORS. Published by Elsevier Inc. on behalf of the Arthroscopy Association of North America. This is an open access article under the CC BY-NC-ND license (http://creativecommons.org/licenses/by-nc-nd/4.0/).

2666-061X/20282

https://doi.org/10.1016/j.asmr.2020.07.014 
reduced ROM during clinical examinations. The anterior impingement examination, i.e., the FADIR (flexionadduction-internal rotation) examination, is positive when patients report pain and there is perceived loss of ROM during a combination of flexion, adduction, and internal rotation. ${ }^{2}$

However, ROM during the FADIR is evaluated qualitatively, and the precise location of impingement between the femur and acetabulum cannot be discerned. Therefore, previous studies have used computer models based on 3-dimensional (3D) reconstructions of bony anatomy segmented from computed tomography (CT) or magnetic resonance imaging data to identify ROM limitations and the location of impingement in an effort to elucidate the pathomechanics of FAI. ${ }^{3-12}$ This technique has been validated to within $5.0 \pm 5.6^{\circ}$ of ROM measured in cadavers using an optoelectronic camera system. ${ }^{11}$ More recently, models have evaluated the extent that ROM is altered by surgical intervention, such as femoral osteochondroplasty for the treatment of cam FAI or rotational acetabular osteotomy for the treatment of acetabular dysplasia., ${ }^{4,6}$

All previous studies that have used ROM simulations of the hip have only evaluated bone-to-bone contact between the femur and the acetabulum. ${ }^{3-12}$ However, use of bone-to-bone contact may oversimplify hip contact mechanics, since these models remove all soft tissue, including the cartilage and labrum. Given the anatomy of the intact, pre-arthritic hip, the femoral neck or articulating surface of the femur would be expected to come into contact with the acetabular cartilage and labrum before there is bone-to-bone collision with the acetabulum. Indeed, using dual-fluoroscopy imaging, previous studies have shown that bone-tobone contact does not occur in vivo during impingement examinations in either asymptomatic participants or patients with cam-type FAI with aspherical femoral anatomy. ${ }^{13,14}$ Thus, inclusion of the anatomy for the acetabular labrum may be required to realistically predict ROM and the location of the impingement site.

The purpose of this study was to determine whether inclusion of the acetabular labrum affected the maximum simulated ROM during the FADIR clinical examination.

\section{Methods}

Asymptomatic participants who had been previously recruited with institutional review board approval for unrelated studies were retrospectively identified. ${ }^{13,15-17}$ Human subjects were included in this study. Our institution provided approval for this study, and informed consent was obtained from all subjects before study participation (institutional review board approvals: University of Utah 10983 and 51053). All research was conducted according to the Declaration of Helsinki. The inclusion criteria were patients who had participated in previous studies with existing anteroposterior screening radiographs and 3D CT arthrogram images available for review and who had no history of hip pain. The exclusion criteria were incomplete imaging and signs of osteoarthritis or hip deformities, including FAI morphology, acetabular dysplasia, acetabular retroversion, or signs of Legg-Calve-Perthes disease or slipped capital femoral epiphysis.

Using a previously published protocol, CT arthrograms of the entire pelvis and proximal femur (1-mm slice thickness) and of the distal femur (3-mm slice thickness) were acquired with variable fields of view for all participants using a Siemens SOMATOM Definition Scanner (Siemens Medical Solutions USA, Inc, Malvern, PA). ${ }^{13,15,16}$ The CT arthrogram provided visualization of the cartilage and labrum of the hip, as well as the bony surfaces of the pelvis and femur (Fig 1A). The femur, pelvis, and acetabular cartilage and labrum were segmented in Amira (6.1; FEI, Hillsboro, OR) to generate 3D surface reconstructions, which were smoothed and decimated for further analysis (Fig 1B).

For consistency across the population and in accordance with previous studies, the neutral pelvic and femoral positions for the ROM simulation were defined from standardized landmarks (Fig 2). ${ }^{8}$ The pelvic coordinate system was constructed using bilateral landmarks of the anterior superior iliac spine (ASIS) and the midpoint of the pubic tubercles to generate the anterior-pelvic plane. The lateral axis of the pelvis connected the 2 ASIS landmarks (Fig 2A), whereas the vertical axis connected the midpoints of the 2 ASIS and the 2 pubic tubercle landmarks (Fig 2B). The femoral coordinate system was constructed from the posterior aspects of the 2 femoral condyles, the knee center, and the femoral head center (FHC) (Fig 2C). The lateral axis of the femur connected the 2 posterior landmarks of the femoral condyles, whereas the vertical axis connected the FHC and the knee center. The neutral position of the hip was defined as the position at which both the pelvic and femoral coordinate systems were aligned rotationally, and the femoral and acetabular centers were aligned. All landmarks were defined using surface-based curvature in PostView (2.4.1; University of Utah, Salt Lake City, UT) and custom scripts in MATLAB (9.3.0; MathWorks, Natick, MA). All rotations were applied about the FHC.

Maximal internal rotation was evaluated at 15 positions defined based on $10^{\circ}$ increments between $70^{\circ}$ and $110^{\circ}$ flexion and $0^{\circ}$ and $20^{\circ}$ adduction to simulate the FADIR examination. For the simulation, the hip was first flexed to the desired angle $\left(70,80,90,100\right.$, or $\left.110^{\circ}\right)($ Fig $3 \mathrm{~A})$ and then adducted $\left(0,10\right.$, or $\left.20^{\circ}\right)$ (Fig $\left.3 \mathrm{~B}\right)$. The femur was then internally rotated until contact occurred, allowing the surfaces to intersect or overlap by up to $0.35 \mathrm{~mm}$ (approximately half the planar width of a single voxel of the CT arthrogram images) to account for minor 


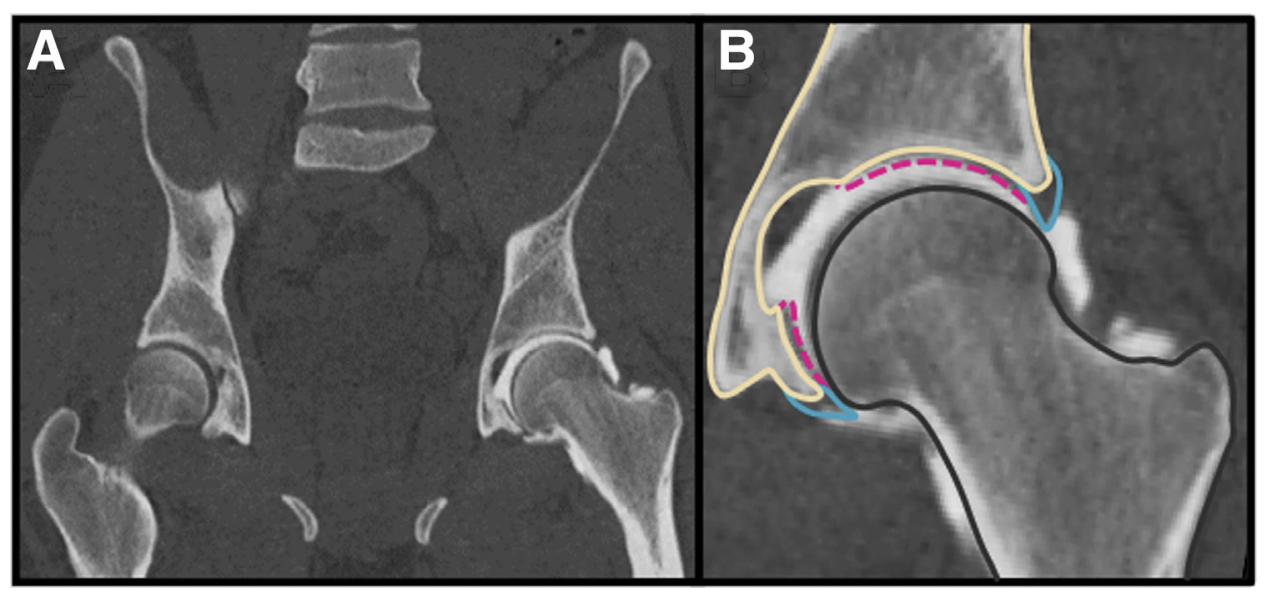

Fig 1. CT arthrogram of the left hip of a representative participant. (A) Radio-opaque contrast agent was injected into one hip of each participant to visualize the cartilage and labrum. (B) Segmentation of the femur (gray), pelvis (tan), and acetabular cartilage (dotted magenta) and labrum (teal) on a single coronal slice from CT. The acetabular cartilage was used to define the center of the acetabulum, but only the surfaces of the femur, pelvis, and labrum were used in the simulations. (CT, computed tomography.)

reconstruction errors of the segmentations (Fig 3C). The simulation was completed to represent 2 contact scenarios for each participant: bone-to-bone contact between the femur and acetabulum (Fig 3D) and bone-to-labrum contact between the femur and labrum (Fig 3E).

The ROM simulation was completed using custom MATLAB scripts, which evaluated surface-based overlap relative to vectors normal to the bone surface. Before the start of the simulation, the pelvis was transformed to represent femoral flexion and adduction while maintaining the femoral coordinate system. For the simulation, the pelvis was fixed and the femur rotated internally in increments of $1^{\circ}$. For larger flexion or adduction angles, the femur may have been in contact with the acetabulum or labrum before any internal rotation; in this scenario, the femur was externally rotated, resulting in a negative internal rotation angle. Due to the morphology of the femur, acetabulum, and labrum (i.e., femoral neck shaft angle, femoral version, and morphology of the anterior inferior iliac spine), a femur position that did not overlap with the pelvis or labrum could not always be found using this simulation protocol. In these cases, no result was recorded for the simulation position for the subject. Similarly, external rotation angles greater than $60^{\circ}$ were considered to be non-physiological and no result was recorded for these cases.

Femoral head and acetabular centers were found by fitting the isolated articulating surface of the femoral head and acetabular cartilage to a 3D ellipsoid, which accounted for the inherent asphericity of the hip joint. The center of the joint was then defined as the center of the fit ellipsoid for the femoral head and the acetabular cartilage. All rotations occurred about the femoral head center, such that the articulating surfaces remained concentric during the simulation. Importantly, the surface of the acetabular cartilage was removed for the simulations, as cartilage contact cannot be properly represented in a simple range of motion simulation.

A clock-face coordinate system was generated from the surface of the acetabular rim and used to describe the position of contact with the femur for both the acetabulum and labrum. The acetabular center was projected onto the best-fit plane of the acetabular rim and represented the center of the clock-face. The clockface was oriented such that 6-o'clock split the acetabular notch, 12-o'clock was directed superiorly, and 3-o'clock was directed anteriorly. ${ }^{18}$ The superior direction of the clock was oriented a mean (standard deviation) of $1.5^{\circ}\left(8.4^{\circ}\right)$ or 0:03 (0:17) posterior from the neutral position used for the simulation.

Values of maximum internal rotation and location of contact relative to the acetabular clock-face for the bone-to-bone and bone-to-labrum simulated contact scenarios were evaluated for normality using the Shapiro-Wilk test. Comparisons between the bone-tobone and bone-to-labrum contact scenarios for each position included all available participants, whereas comparisons between simulation positions only included the participants who were available for both positions for the specific contact scenario. Nonnormally distributed data were evaluated using the Wilcoxon rank sum test and are presented as median [25th percentile, 75 th percentile]. Normally distributed data and demographics are presented as mean (standard deviation) and evaluated with the Student $t$ test. All statistical analyses were completed in $\mathrm{R}$ (3.6.1; R Core Team, Vienna, Austria). 

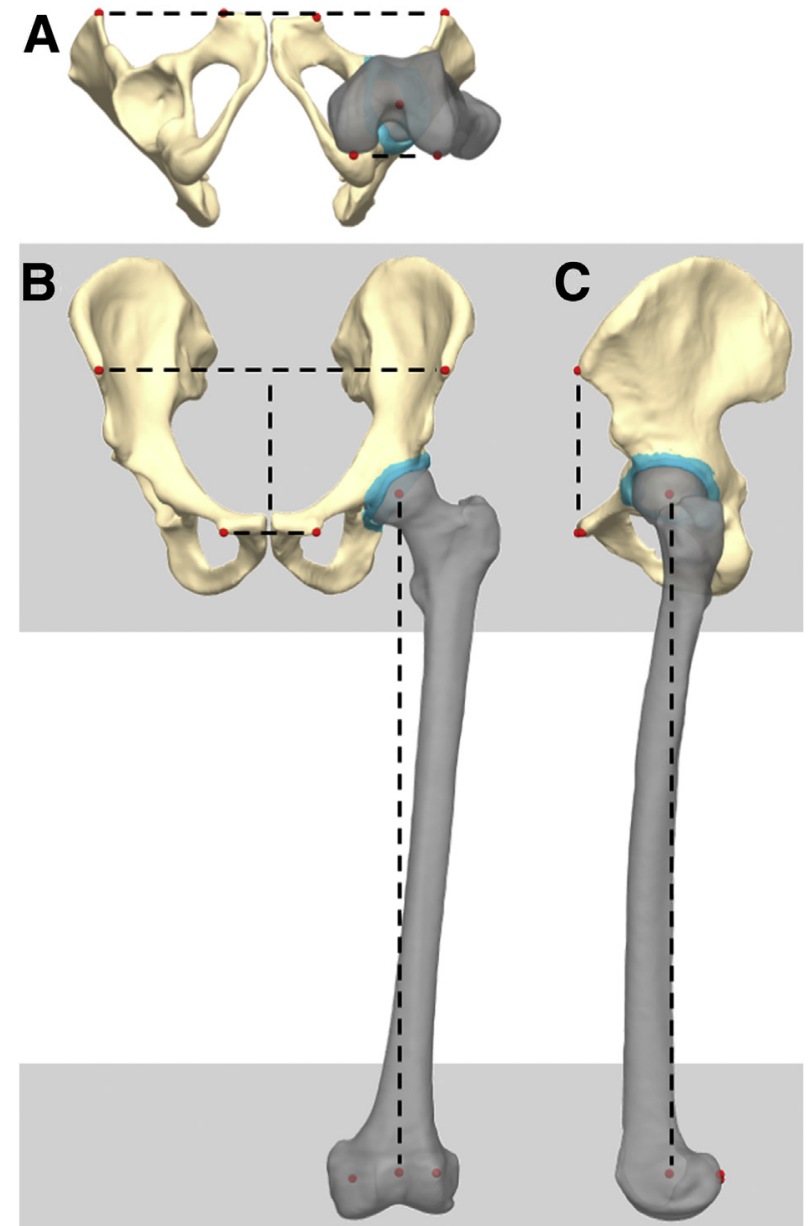

Fig 2. A representative pelvis (tan), left femur (semi-transparent gray), and labrum (semi-transparent teal) in neutral orientation displaying the landmarks and reference coordinate systems from an (A) inferior, (B) anterior, and (C) left view. Coordinate system axis definitions are shown with dotted black lines. The pelvic reference was defined from both ASIS and the midpoint of the pubic tubercle landmarks (red). The femoral reference was defined from the femoral head center, the knee center, and the posterior aspect of the femoral condyles landmarks (red). (ASIS, anterior superior iliac spine.)

\section{Results}

Nineteen participants ( 9 female) had a mean age of 25 (3) years and body mass index of 21.3 (2.8). From the previously published datasets, 2 participants were excluded due to incomplete imaging of the distal femur, such that the femoral coordinate system could not be generated, and 3 were excluded due to initial penetration between the femoral head and labrum in all simulation positions (2 participants) or all simulation positions which included non-neutral adduction (1 participant). The simulation resulted in a feasible FADIR examination position for at least 17 of the 19 participants for each of the 15 simulation positions for the bone-to-bone contact scenario and 10 of 19 for the bone-to-labrum contact scenario (Table 1).
The angle of maximum internal rotation for the femur-labrum contact model was significantly smaller than that of the femur-acetabulum contact model for all participants and all positions $(P \leq .001$ for all), except at $110^{\circ}$ flexion and $20^{\circ}$ adduction $(P=.114$; Table 2$)$. The inclusion of the labrum resulted in a reduction of internal rotation ROM of $18[15,25]^{\circ}$ when compared with the bone-to-bone simulation (Fig 4).

The median reduction in simulated ROM due to altered flexion angle was generally greater for the bone-to-labrum contact scenario (range, $-7^{\circ}$ to $-12^{\circ}$ ) in comparison with the bone-to-bone contact scenario (range, $-6^{\circ}$ to $-9^{\circ}$; Table 3 ), especially at larger flexion angles. A similar observation was made for altered adduction angles; however, the magnitude of the changes was smaller (range, $-3^{\circ}$ to $0^{\circ}$; Table 3 ).

The location of impingement only varied by $-0: 01$ [-0:27, 0:16] between the bone-to-bone and bone-tolabrum contact scenarios; however, the bone-to-labrum contact scenario resulted in nearly double the variability across participants, as indicated by interquartile range (Table 4). The median acetabular clock-face position of contact varied by $0: 12$ or less for all $10^{\circ}$ increments of adduction and flexion between the bone-to-bone (range, $-0: 09$ to 0:05) and bone-to-labrum (range, $-0: 05$ to $0: 12$ ) contact scenarios. The contact location was more inferior for the bone-to-labrum contact model than the bone-to-bone contact model at $100^{\circ}$ flexion and $0^{\circ}$ adduction $(P=.012)$ and at $110^{\circ}$ flexion and $0^{\circ}$ adduction $(P=.003$; Fig 5$)$.

\section{Discussion}

Inclusion of the anatomy for the acetabular labrum significantly reduced simulated hip ROM, with a reduction in maximum $\mathrm{ROM}$ of approximately $20^{\circ}$ internal rotation for all evaluated positions between $70^{\circ}$ and $110^{\circ}$ flexion and $0^{\circ}$ and $20^{\circ}$ adduction when compared with the bone-to-bone simulation. Although it is known that the bony anatomy of the femur and acetabulum do not come into contact in vivo, even during large ROM activities, ${ }^{13,14}$ computer simulation studies, which assume hip ROM limitations due to direct bone-to-bone contact, are likely still useful in identifying abnormal bony morphology that causes impingement. ${ }^{3-11}$ As such, it is relevant to compare our ROM for the bone-to-bone contact scenario with those of previous studies. A number of previous studies have evaluated the FADIR examination with varying degrees of flexion and adduction using ROM simulation in control participants, as well as patients, such as those

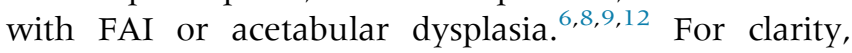
comparison with only the results from the control cohorts are included herein. The internal rotation achieved in control subjects by Kubiak-Langer et al. ${ }^{8}$ was reduced when compared with the angles we observed by approximately $10-15^{\circ}$ for all simulation 

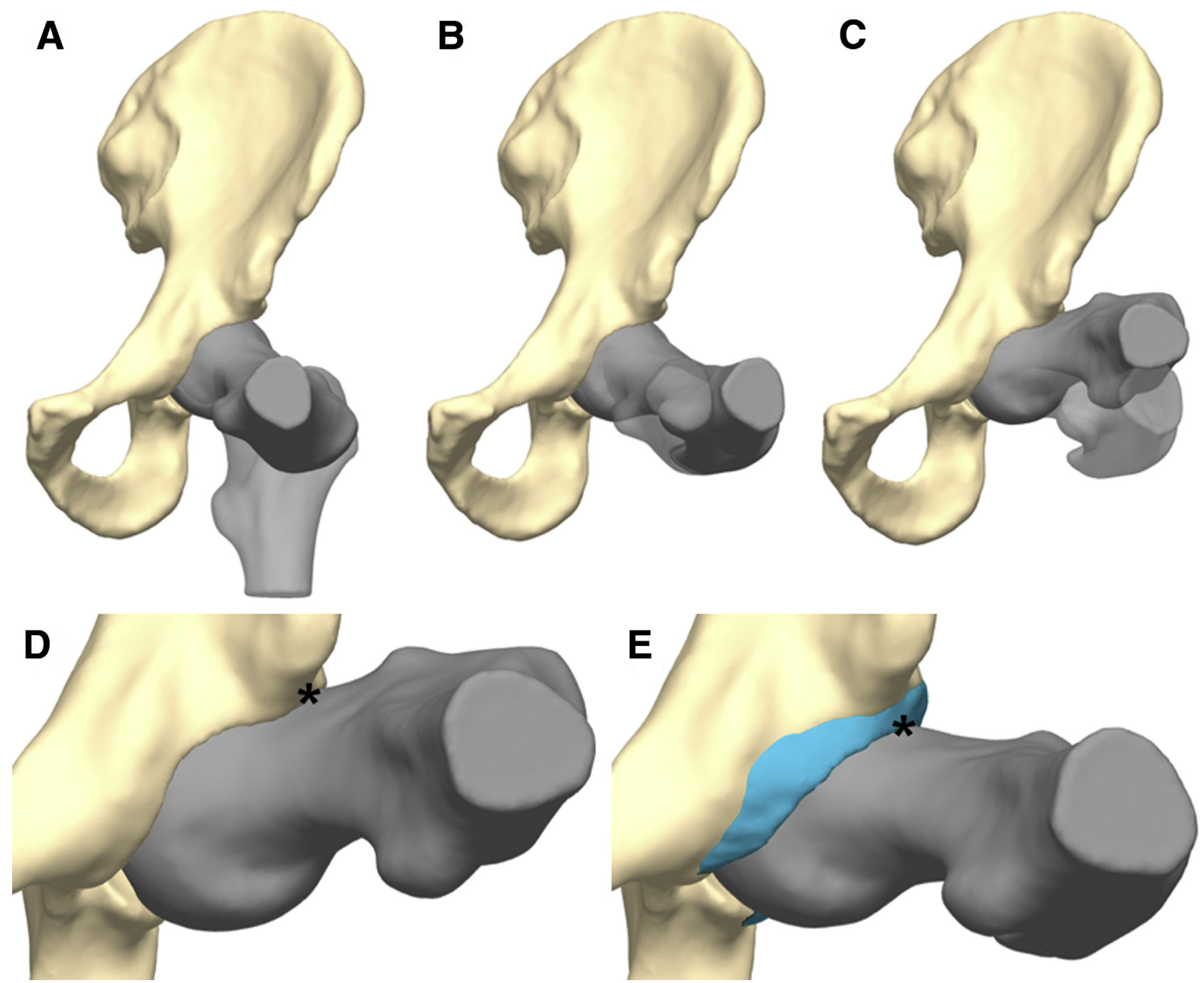

Fig 3. Anterior view of left hemipelvis and proximal femur models for a representative participant during simulated $90^{\circ}$ flexion, $20^{\circ}$ adduction, and internal rotation to contact. Semitransparent femurs represent the initial position, whereas the opaque femur represents the transformed position. (A) From a neutral orientation the femur is flexed to $90^{\circ}$, (B) adducted to $20^{\circ}$, (C) and internally rotated until the femur contacts either the bony surface of the acetabulum or soft-tissue surface of the labrum. The region of contact between the femur and the (D) acetabulum and (E) labrum is shown with an asterisk (*).

positions (see Fig 4A in Kubiak-Langer et al., whereas in Table 2, the ROM is within $5^{\circ}$ of internal rotation for $0^{\circ}$ adduction, $90^{\circ}$ flexion). However, the maximum internal rotation ROM we observed was approximately
10- $15^{\circ}$ less than that reported by Nakahara et al. for $0^{\circ}$ adduction and $0-15^{\circ}$ larger for the $20^{\circ}$ adduction scenario for flexion angles between $80^{\circ}$ and $110^{\circ}$ with differences increasing with flexion (see Fig 4 in

Table 1. The Number of Computer Simulation Results Included in the Analysis of Range of Motion for Each Position and Contact Scenario

\begin{tabular}{|c|c|c|c|c|c|c|}
\hline & \multicolumn{2}{|c|}{$0^{\circ}$ Adduction } & \multicolumn{2}{|c|}{$10^{\circ}$ Adduction } & \multicolumn{2}{|c|}{$20^{\circ}$ Adduction } \\
\hline$\overline{70^{\circ} \text { flexion }}$ & $\mathrm{n}=19$ & $\mathrm{n}=19$ & $\mathrm{n}=19$ & $\mathrm{n}=19$ & $\mathrm{n}=19$ & $\mathrm{n}=19$ \\
\hline $90^{\circ}$ flexion & $\mathrm{n}=19$ & $\mathrm{n}=19$ & $\mathrm{n}=19$ & $\mathrm{n}=19$ & $\mathrm{n}=19$ & $\mathrm{n}=19$ \\
\hline $100^{\circ}$ flexion & $\mathrm{n}=19$ & $\mathrm{n}=19$ & $\mathrm{n}=19$ & $\mathrm{n}=18$ & $\mathrm{n}=18$ & $\mathrm{n}=13$ \\
\hline $110^{\circ}$ flexion & $\mathrm{n}=19$ & $\mathrm{n}=15$ & $\mathrm{n}=19$ & $\mathrm{n}=16$ & $\mathrm{n}=17$ & $\mathrm{n}=10$ \\
\hline
\end{tabular}

NOTE: Simulations were not completed when rotation of the femur could not reduce overlap between the femur and acetabulum or labrum to less than $0.35 \mathrm{~mm}$, the approximate voxel half-width of the acquired computed tomography images. 
Table 2. Maximum Internal Rotation for Each FADIR Examination Simulation and Contact Scenario

\begin{tabular}{|c|c|c|c|c|c|c|}
\hline & \multicolumn{2}{|c|}{$0^{\circ}$ Adduction } & \multicolumn{2}{|c|}{$10^{\circ}$ Adduction } & \multicolumn{2}{|c|}{$20^{\circ}$ Adduction } \\
\hline$\overline{70^{\circ} \text { flexion }}$ & $48^{\circ}[46,55]^{\circ}$ & $33^{\circ}[30,39]^{\circ}$ & $50^{\circ}[46.5,56]^{\circ}$ & $32^{\circ}[28.5,39.5]^{\circ}$ & $49^{\circ}[44.5,55]^{\circ}$ & $28^{\circ}[23,34.5]^{\circ}$ \\
\hline $90^{\circ}$ flexion & $38^{\circ}[34.5,41.5]^{\circ}$ & $19^{\circ}[15.5,24]^{\circ}$ & $38^{\circ}[33.5,41.5]^{\circ}$ & $18^{\circ}[13.5,25]^{\circ}$ & $36^{\circ}[30,41.5]^{\circ}$ & $16^{\circ}[5.5,21.5]^{\circ}$ \\
\hline $100^{\circ}$ flexion & $33^{\circ}[27,35]^{\circ}$ & $9^{\circ}[4.5,16]^{\circ}$ & $31^{\circ}[25,37]^{\circ}$ & $13.5^{\circ}[7.5,16.75]^{\circ}$ & $32^{\circ}[20,38.75]^{\circ}$ & $15^{\circ}[-1,19]^{\circ}$ \\
\hline $110^{\circ}$ flexion & $23^{\circ}[17.5,28]^{\circ}$ & $1^{\circ}[-7.5,11]^{\circ}$ & $22^{\circ}[14,32]^{\circ}$ & $-2^{\circ}[-8.75,3]^{\circ}$ & $27^{\circ}[9,39]^{\circ}$ & $12^{\circ}[10.25,17]^{\circ}$ \\
\hline
\end{tabular}

NOTE: Data presented as median [interquartile range].

FADIR, flexion-adduction-internal rotation.

Nakahara et al.). ${ }^{9}$ Lerch et al. evaluated internal rotation at $0^{\circ}$ and $10^{\circ}$ adduction between $90^{\circ}$ and $120^{\circ}$ flexion and reported values that appear to be within approximately $5^{\circ}$ of those found herein (see Fig 4, A and $\mathrm{B}$ in Lerch et al.). ${ }^{12}$ Relative to the 3 adduction angles at $90^{\circ}$ flexion, our bone-to-bone simulations resulted in $11-17^{\circ}$ less internal rotation than that measured by Iwai et al. ${ }^{6}$ Our results agree with previous studies evaluating internal rotation at $90^{\circ}$ flexion, which found maximum internal rotation to occur between $35^{\circ}$ and $50^{\circ}$ for control populations $\left(35^{\circ}\right.$ by Kubiak-Langer et al., $35^{\circ}$ by Tannast et al., $44^{\circ}$ and $50^{\circ}$ by Nakahara et al., and $40^{\circ}$ by Iwai et al.)..$^{6,8-11}$ As observed in previous studies, the maximum internal rotation ROM decreased with increased flexion and adduction; however, our results did not indicate as large an effect due to adduction as that observed by Nakahara et al. ${ }^{9,10}$

Few studies have evaluated the location of contact between the femur and acetabulum using ROM simulations. However, our results align well with the simulations of Kubiak-Langer et al., who observed the greatest frequency of contact location at 1:45 based on the clock-face developed from the anterior pelvic plane. ${ }^{8}$ The slightly more superior location of contact in the current study may be a result of the adjustment of the clock-face to align with the acetabular notch.

Similar to other studies, we used the anterior pelvic plane, which aligned the bilateral ASIS landmarks and the midpoint between the 2 pubic tubercle landmarks with the anterior plane..$^{8,10,11}$ However, other studies have used the functional pelvic plane, which accounts for anteroposterior tilt of the pelvis while supine, ${ }^{5-7,9,10}$ or the International Society of Biomechanics coordinate system, which aligns the 2 ASIS landmarks with the midpoint of the 2 posterior superior iliac spine landmarks with the axial plane. ${ }^{3,19}$ With 106 participants, Nakahara et al. found that use of the anterior pelvic plane resulted in an average of $1.8^{\circ}$ decrease in internal rotation at $90^{\circ}$ flexion compared with the functional pelvic plane (mean $3.8^{\circ}$ pelvic tilt). ${ }^{10}$ The effect of pelvic coordinate systems is therefore approximately an
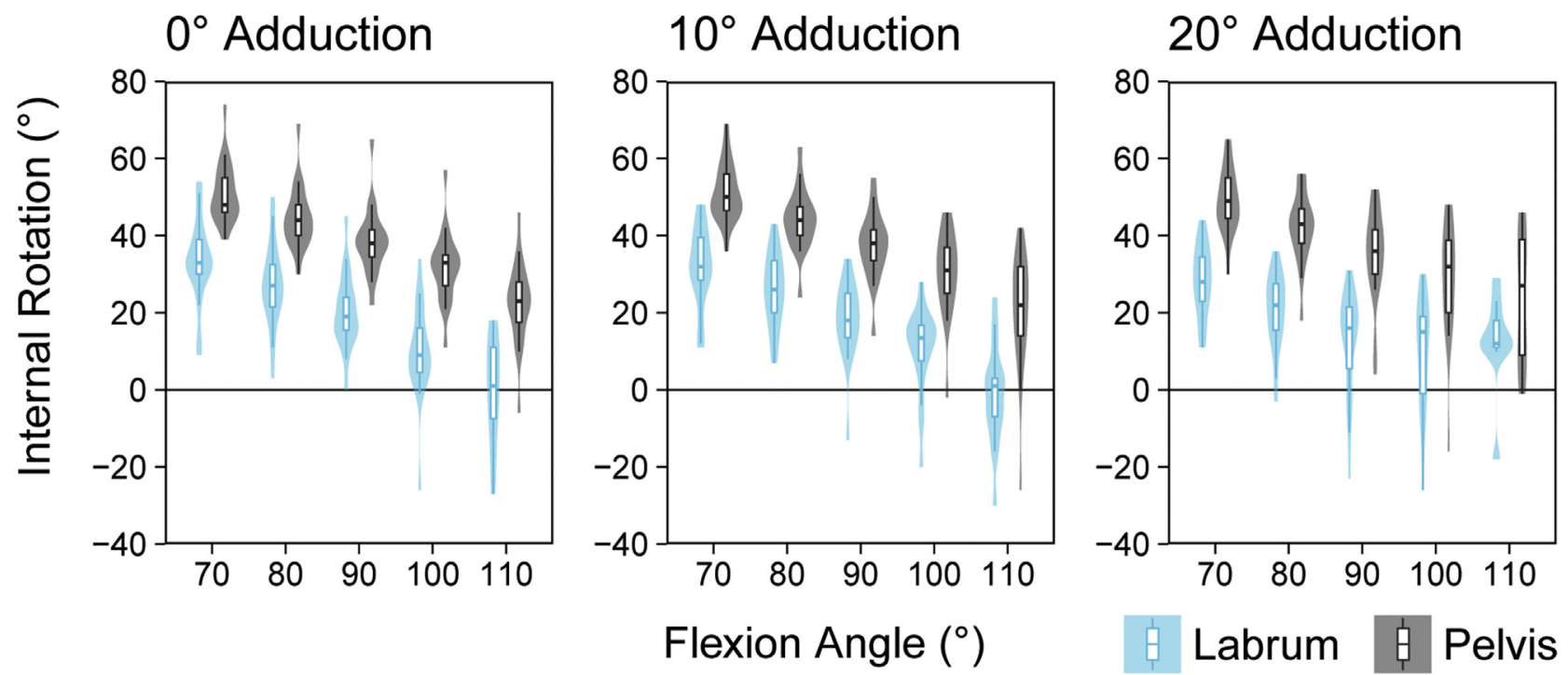

Fig 4. Maximum internal rotation angle for range of motion simulations for the femur-acetabulum and femur-labrum scenarios. Each plot represents a specific angle of adduction and each of the associated flexion angles. Boxplot represents the median and interquartile range, whereas the vertical lines indicate 1.5 times the interquartile range. Semitransparent violin plot displays the distribution of the internal rotation values for each simulation. 
order of magnitude less than what we observed for the effect of inclusion of the labrum and would not be expected to alter our conclusions.

Kapron et al. ${ }^{14}$ measured in vivo ROM during the FADIR examination using dual-fluoroscopy and observed a mean position of $93^{\circ}$ flexion, $11^{\circ}$ adduction, and $19^{\circ}$ internal rotation for 6 asymptomatic control participants. Data reported by Kapron et al. inherently included the contribution of the acetabular labrum, since this structure was intact in the participants analyzed in their study. In our study, the labrum was considered, but hip motion and contact was instead simulated using bone and labrum surface reconstructions, not measured in vivo. Still, we found that at $90^{\circ}$ flexion and $10^{\circ}$ adduction the average femoral internal rotation to engage contact with the labrum was $18[13.5,25]^{\circ}$, which aligns well with data reported by Kapron et al.

Considering that patients often present with labral damage during clinical evaluation for FAI or acetabular dysplasia, improved understanding of the interaction between the femur and labrum during high ROM activities may be crucial to our understanding of these pathologies. Although previous simulation studies have helped to identify gross morphologic changes that cause early abutment between the femoral head and acetabular labrum, ${ }^{3-11}$ they represent an unlikely scenario in which bone-to-bone contact exists within the hip. Nevertheless, bone-to-bone contact simulations may be informative in the evaluation of the relative change in ROM on the basis of a surgical intervention (e.g., before and after femoral osteochondroplasty for patients with FAI or periacetabular osteotomy for acetabular dysplasia patients) when the region of contact is considered. ${ }^{4-6,8,9}$ Therefore, the effect of the labrum on intra-articular impingement evaluated herein may be useful in interpretation of the errors in estimation of impingement and ROM using kinematic simulations of the hip.

\section{Limitations}

We note several limitations to our study, many of which are limitations inherent to ROM simulations. First, since the simulation defines the end ROM by contact between 2 surfaces, it is not possible to include cartilage surfaces as cartilage contact occurs throughout motion. Second, simulations may not accurately represent active or passive hip ROM in vivo. Herein, we simulated internal rotation ROM of the femur for FADIR examination but do not evaluate either active or passive ROM for these patients. We chose to simulate this clinical examination, as it provides important insight on the effect of the anatomy of the anterior labrum on ROM, where the anatomy of the labrum is more variable. ${ }^{18}$ Third, motion of the pelvis was not considered, yet a previous dual-fluoroscopy imaging 
Table 4. Acetabular Clock-Face Position of Contact at Maximum Internal Rotation for Each FADIR Examination Simulation and Contact Scenario

\begin{tabular}{|c|c|c|c|c|c|c|}
\hline & \multicolumn{2}{|c|}{$0^{\circ}$ Adduction } & \multicolumn{2}{|c|}{$10^{\circ}$ Adduction } & \multicolumn{2}{|c|}{$20^{\circ}$ Adduction } \\
\hline $70^{\circ}$ flexion & $1: 29[1: 17,1: 37]$ & $1: 18[0: 53,1: 32]$ & $1: 23[1: 19,1: 48]$ & $1: 18[0: 35,1: 44]$ & $1: 14[1: 05,1: 58]$ & $1: 52[0: 46,2: 19]$ \\
\hline $90^{\circ}$ flexion & $1: 18[1: 10,1: 28]$ & $1: 19[1: 09,1: 36]$ & $1: 12[0: 53,1: 37]$ & $1: 17[0: 34,1: 44]$ & $1: 14[0: 34,2: 00]$ & $2: 03[0: 17,2: 39]$ \\
\hline $100^{\circ}$ flexion & $1: 19[1: 12,1: 37]$ & $1: 49[1: 21,1: 58]$ & $1: 23[0: 52,1: 53]$ & $1: 25[0: 39,1: 59]$ & $1: 07[0: 26,1: 59]$ & $0: 29[0: 09,2: 30]$ \\
\hline $110^{\circ}$ Flexion & $1: 28[1: 15,1: 36]$ & $1: 48[1: 38,1: 56]$ & $1: 19[0: 55,1: 56]$ & $2: 01[1: 21,2: 28]$ & $1: 02[0: 22,2: 11]$ & $0: 19[-0: 04,0: 58]$ \\
\hline
\end{tabular}

NOTE: Data presented as median [interquartile range] on the clock-face.

FADIR, flexion-adduction-internal rotation.

study found that the pelvis moves substantially once the labrum is contacted during the FADIR examination. ${ }^{14}$ Simulation including a stationary pelvis is, however, the standard protocol for computational simulations of hip ROM.

There are also limitations specific to our study. Herein, we assumed that maximum ROM of the hip occurred upon contact of the femur with the acetabulum or labrum. This assumption is valid for bone-tobone contact simulations, as cortical bone is very stiff; however, the labrum and underlying cartilage would likely conform to the femur allowing for additional motion due to compression or conformation of the soft tissue. ${ }^{14}$ Our study is also unable to quantify the amount of motion that would occur due to conformation of the labrum to the femur nor the amount of motion that would eventually lead to labral tears or chondral pathology. A related concern is that of the morphology of the labrum; to provide clear visualization of the labrum and separate cartilage layers of the femur and acetabulum, traction was applied during image acquisition. However, as the labrum is marginal to the hip capsule, the increased tension on the capsule may have altered the morphology of the labrum and influenced the observed results. While the labrum might become inverted with large amounts of traction, the amount of traction used herein aimed only to separate the acetabular and femoral cartilage surfaces and does not appear to have deformed the labrum (Fig 1). Finally, our study only used images from 19 participants to develop the computer simulations. This reduces the ability to generalize the effect of the labrum in symptomatic individuals.

\section{Conclusions}

Inclusion of the anatomy of the acetabular labrum in collision models used to simulate impingement examinations reduced the internal rotation ROM by approximately $20^{\circ}$ and increased variability in the location of contact relative to the acetabular rim. $0^{\circ}$ Adduction

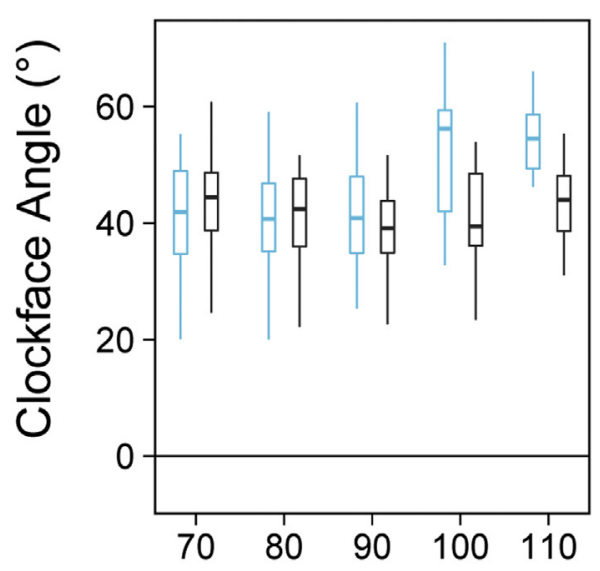

$10^{\circ}$ Adduction

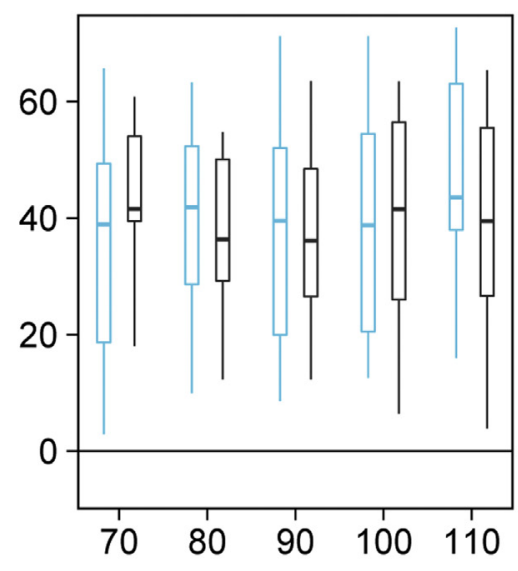

Flexion Angle $\left({ }^{\circ}\right)$ $20^{\circ}$ Adduction

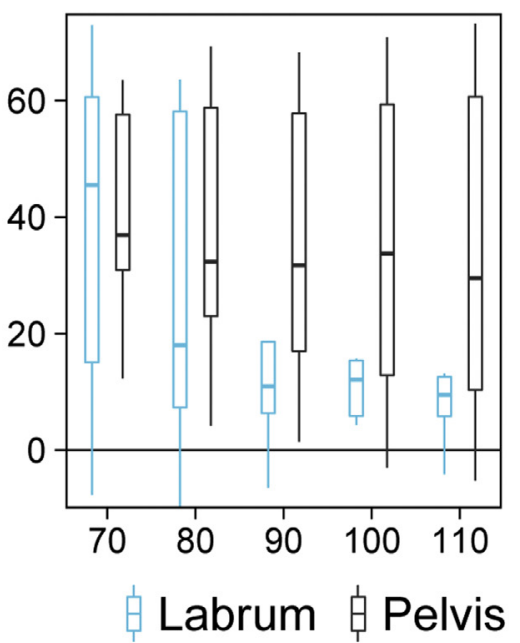

Fig 5. Maximum internal rotation angle for range of motion simulations for the femur-acetabulum and femur-labrum scenarios. Each plot represents a specific angle of adduction and each of the associated flexion angles. Boxplot represents the median and interquartile range, whereas the vertical lines indicate 1.5 times the interquartile range. 


\section{References}

1. Ganz R, Parvizi J, Beck M, Leunig M, Notzli H, Siebenrock KA. Femoroacetabular impingement: A cause for osteoarthritis of the hip. Clin Orthop Rel Res 2003:112-120.

2. Klaue K, Durnin CW, Ganz R. The acetabular rim syndrome. A clinical presentation of dysplasia of the hip. J Bone Joint Surg Br 1991;73:423-429.

3. Audenaert EA, Mahieu P, Pattyn C. Three-dimensional assessment of cam engagement in femoroacetabular impingement. Arthroscopy 2011;27:167-171.

4. Bedi A, Dolan M, Magennis E, Lipman J, Buly R, Kelly BT. Computer-assisted modeling of osseous impingement and resection in femoroacetabular impingement. Arthroscopy 2012;28:204-210.

5. Hamada H, Takao M, Nakahara I, Sakai T, Nishii T, Sugano N. Hip range-of-motion (ROM) is less than normal after rotational acetabular osteotomy for developmental dysplasia of the hip: A simulated ROM analysis. J Orthop Res 2016;34:217-223.

6. Iwai S, Kabata T, Maeda T, et al. Three-dimensional kinetic simulation before and after rotational acetabular osteotomy. J Orthop Sci 2014;19:443-450.

7. Kobayashi N, Inaba Y, Kubota S, et al. The distribution of impingement region in cam-type femoroacetabular impingement and borderline dysplasia of the hip with or without cam deformity: A computer simulation study. Arthroscopy 2017;33:329-334.

8. Kubiak-Langer M, Tannast M, Murphy SB, Siebenrock KA, Langlotz F. Range of motion in anterior femoroacetabular impingement. Clin Orthop Rel Res 2007;458:117-124.

9. Nakahara I, Takao M, Sakai T, Miki H, Nishii T, Sugano N. Three-dimensional morphology and bony range of movement in hip joints in patients with hip dysplasia. Bone Joint J 2014;96-b:580-589.

10. Nakahara I, Takao M, Sakai T, Nishii T, Yoshikawa H, Sugano N. Gender differences in 3D morphology and bony impingement of human hips. J Orthop Res 2011 ;29:333-339.

11. Tannast M, Kubiak-Langer M, Langlotz F, Puls M, Murphy SB, Siebenrock KA. Noninvasive three-dimensional assessment of femoroacetabular impingement. J Orthop Res 2007;25:122-131.

12. Lerch TD, Degonda C, Schmaranzer F, et al. Patient-specific 3-D magnetic resonance imaging-based dynamic simulation of hip impingement and range of motion can replace 3-d computed tomography-based simulation for patients with femoroacetabular impingement: Implications for planning open hip preservation surgery and hip arthroscopy. Am J Sports Med 2019;47:2966-2977.

13. Kapron AL, Aoki SK, Peters CL, Anderson AE. Subjectspecific patterns of femur-labrum contact are complex and vary in asymptomatic hips and hips with femoroacetabular impingement. Clin Orthop Rel Res 2014;472:3912-3922.

14. Kapron AL, Aoki SK, Peters CL, Anderson AE. In-vivo hip arthrokinematics during supine clinical exams: Application to the study of femoroacetabular impingement. J Biomech 2015;48:2879-2886.

15. Henak CR, Abraham CL, Peters CL, Sanders RK, Weiss JA, Anderson AE. Computed tomography arthrography with traction in the human hip for three-dimensional reconstruction of cartilage and the acetabular labrum. Clin Radiol 2014;69:e381-e391.

16. Fiorentino NM, Kutschke MJ, Atkins PR, Foreman KB, Kapron AL, Anderson AE. Accuracy of functional and predictive methods to calculate the hip joint center in young non-pathologic asymptomatic adults with dual fluoroscopy as a reference standard. Ann Biomed Engin 2016;44:2168-2180.

17. Henak CR, Ellis BJ, Harris MD, Anderson AE, Peters CL, Weiss JA. Role of the acetabular labrum in load support across the hip joint. J Biomech 2011;44:2201-2206.

18. Philippon MJ, Michalski MP, Campbell KJ, et al. An anatomical study of the acetabulum with clinical applications to hip arthroscopy. J Bone Joint Surg Am 2014;96: 1673-1682.

19. Wu G, Siegler S, Allard P, et al. ISB recommendation on definitions of joint coordinate system of various joints for the reporting of human joint motion-part I: Ankle, hip, and spine. International Society of Biomechanics. J Biomech 2002;35:543-548. 